\section{Criterios exigidos en revistas odontológicas venezolanas para la publicación de la fotografía extraoral en casos clínicos}

\section{Criteria required in Venezuelan dental journals for the publication of the extraoral photographs in case reports}

\begin{abstract}
Resumen
Objetivo. Describir los criterios exigidos en revistas odontológicas venezolanas para la publicación de la fotografía clínica extraoral en los casos clínicos publicados en el periodo 2010-2021. Métodos. Investigación de enfoque cuantitativo, alcance descriptivo y transversal. Se analizaron artículos científicos que incluyeran fotografías clínicas extraorales publicadas en revistas odontológicas venezolanas entre 2010 y 2021. Resultados. Se observó exigencia por parte de la editorial en las estrategias de preservación de identidad del paciente, sumado al uso del consentimiento informado para las publicaciones de fotografías clínicas extraorales de casos clínicos. Conclusiones. La revista Acta Odontológica hace mayor uso de consentimiento informado, además de utilizar el enmascaramiento y anonimato como método de preservación de la identidad en sus publicaciones.
\end{abstract}

Palabras clave: Fotografía; Fotografía dental; Consentimiento informado; Ética en la publicación científica; Ética odontológica (fuente: DeCS BIREME).

\begin{abstract}
Objective. To describe the criteria required in Venezuelan dental journals for the publication of extraoral clinical photography in clinical cases published in the period 20102021. Methods. Research with a quantitative approach, descriptive scope, and cross-sectional design. Scientific articles that included extraoral clinical photographs published in Venezuelan dental journals between 2010 and 2021 were analyzed. Results. There was a need from the publisher in the strategies of preservation of the patient's identity, and to the use of informed consent for the publication of photographs. extraoral clinics of clinical cases. Conclusions. The Acta Odontológica journal makes greater use of informed consent, in addition to using masking and anonymity as a method of identity preservation in its publications.
\end{abstract}

Keywords: Photography; Dental photography; Informed consent; Scientific publication ethics; Dental ethics (source: MeSH NLM).

\section{Artículo Original}

Airin Avendaño ${ }^{1, a}$, Karen Avendaño 1,a, Darío Sosa 1,b

${ }^{1}$ Universidad de Los Andes, Facultad de Odontología, Mérida, Venezuela.

a Estudiante de Pregrado.

${ }^{\mathrm{b}}$ Magíster en Etnología, mención Etnohistoria.

\section{Correspondencia:}

Airin Avendaño: airinrondon@gmail.com Facultad de Odontología. Edificio Rectorado, Departamento de Investigación. Calle 24, Centro. Mérida, Venezuela

ORCID: 0000-0002-3185-9622

\section{Coautores:}

Karen Avendaño: karen.avendano31@gmail.com

ORCID: 0000-0001-9531-5133

Darío Sosa: dario.sosa@gmail.com

ORCID: 0000-0001-6202-097X

\section{Editora:}

Rosse Mary Falcón-Antenucci

Universidad Inca Garcilaso de la Vega, Perú

Conflicto de intereses: los autores declaran no tener conflictos de interés.

Fuente de financiamiento: autofinanciado.

Recibido: $12 / 09 / 21$

Aceptado: 30/09/21

Publicado: $21 / 01 / 22$ 


\section{Introducción}

El uso de la fotografía clínica brinda al profesional de la salud una herramienta extraordinaria, debido a la versatilidad para la elaboración y el esclarecimiento de los planes de tratamiento. Un ejemplo claro de ello es la fotografía extraoral, pues representa una herramienta importante para el análisis de patrones faciales ${ }^{1-3}$, facilitando la evaluación de resultados ${ }^{4} \mathrm{e}$ interpretación de detalles que pueden pasar desapercibidos durante el examen clínico por parte de ortodoncistas, rehabilitadores, esteticistas, cirujanos maxilo-faciales y cirujanos plásticos ${ }^{1,5,6}$. Así como también mejora, facilita y complementa la comunicación entre el paciente y el profesional mediante imágenes que ilustran los tratamientos a realizar ${ }^{7-9}$, sirve como medio de motivación y educación al paciente ${ }^{10,11}$, y de la misma forma, suministra un método objetivo ante la comunicación con otros profesionales y con el laboratorio dental y laboratorio histopatológico ${ }^{12}$, tomando en cuenta el rumbo que ha tomado la odontología en el mundo digital.

Por otra parte, debido a la creciente demanda de tratamientos odontológicos, cada día es más importante la obtención de registros fotográficos que permitan una evaluación objetiva de los resultados, proporcionen una herramienta de presentación en congresos y artículos ${ }^{11,13}$, lo que contribuye con el crecimiento de la documentación fotográfica científica con altos estándares de calidad, pues serán utilizados para la divulgación científica y la enseñanza en universidades.

Otro de los usos de la fotografía clínica está ligado a respaldar la documentación médico-legal pues estará disponible en caso de litigio, la imagen de las condiciones del paciente antes, durante y después del tratamiento, tomando en cuenta que cualquier acto odontológico impartido representa una responsabilidad legal ${ }^{14-16}$.

La fotografía clínica, al ser realizada de forma sistemática, satisface plenamente su finalidad de documentación científica, principalmente en relación a su reproductibilidad ${ }^{17,18}$. Dicha documentación deberá realizarse de forma adecuada, y en caso que el profesional decida hacer uso de las fotografías para publicación es importante que tenga en cuenta los criterios apropiados para llevarla a cabo ${ }^{19,20}$, con el fin de evitar un análisis incorrecto de las mismas ${ }^{1}$. En este sentido, la fotografía divulgada será completa responsabilidad del autor del trabajo, pues es un requisito para algunas publicaciones científicas, siendo así un valioso instrumento para la Odontología ${ }^{21}$.

Principalmente en fotografías extraorales, se hace necesario el empleo de permisos por escrito para la realización, y uso específico de las mismas, así como, usualmente, se emplea el uso de estrategias generales para proteger la privacidad del paciente al momento de publicar dichas fotografías; sin embargo, estos métodos han sido poco eficaces y existe discordancia de criterios al momento de recomendar el enmascaramiento facial para mantener el anonimato del paciente ya que afectan directamente la estética y visualización adecuada de la fotografía extraoral ${ }^{21}$.
De este modo, diferentes artículos afirman que no es necesario el enmascaramiento siempre y cuando se cuente con la autorización para la toma y divulgación de fotografías extraorales en el consentimiento informado ${ }^{22-27}$. Esta autorización debe estar elaborada correctamente con el fin de proteger al profesional y al paciente de cualquier problema a futuro en cuanto al uso de la imagen ${ }^{20} \mathrm{y}$ ejecución de procedimientos clínicos ${ }^{28}$.

Finalmente, se han realizado estudios que han presentado información acerca de técnicas que permitan el correcto desarrollo de fotografías intraorales y extraorales, su importancia, consejos sobre los equipos más pertinentes para el correcto desarrollo de las mismas, e incluso, se han propuesto parámetros por medio de los cuales se ha sistematizado la presentación de la fotografía clínica extraoral 2,3,14. Asimismo, algunos estudios han publicado información sobre el uso incorrecto del consentimiento informado, o la ausencia total del mismo ${ }^{29}$.

La fotografía clínica, al ser realizada de forma sistemática, satisface plenamente su finalidad de documentación científica, principalmente en relación a su reproductibilidad ${ }^{17,18}$. Dicha documentación deberá realizarse de forma adecuada, y en caso que el profesional decida hacer uso de las fotografías para publicación es importante que tenga en cuenta los criterios apropiados para llevarla a cabo ${ }^{19,20}$, con el fin de evitar un análisis incorrecto de las mismas ${ }^{1}$. En este sentido, la fotografía divulgada será completa responsabilidad del autor del trabajo, pues es un requisito para algunas publicaciones científicas, siendo así un valioso instrumento para la Odontología ${ }^{18}$.

Usualmente se emplea el uso de estrategias generales para proteger la privacidad del paciente al momento de publicar dichas fotografías, sin embargo, estos métodos han sido poco eficaces y existe discordancia de criterios al momento de recomendar el enmascaramiento facial para mantener el anonimato del paciente ya que afectan directamente la estética y visualización adecuada de la fotografía extraoral ${ }^{21}$.

De este modo, diferentes artículos afirman que no es necesario el enmascaramiento siempre y cuando se cuente con la autorización para la toma y divulgación de fotografías extraorales en el consentimiento informado. Esta autorización debe estar elaborada correctamente con el fin de proteger al profesional y al paciente de cualquier problema a futuro en cuanto al uso de la imagen ${ }^{20} \mathrm{y}$ ejecución de procedimientos clínicos ${ }^{28}$.

A pesar que se han realizado estudios acerca de las políticas editoriales de revistas científicas alrededor del mundo en lo que a divulgación de fotografías extraorales se refiere ${ }^{22,30-38}$ no se han encontrado estudios donde se analicen los criterios de publicación de la fotografía clínica extraoral en las revistas odontológicas venezolanas. Siendo la fotografía clínica una herramienta esencial para el desarrollo de la práctica odontológica diaria y la divulgación de la información, la presente investigación tiene como objetivo describir los criterios exigidos en revistas odontológicas venezolanas para la publicación 
de la fotografía clínica extraoral en los casos clínicos publicados en el periodo 2010-2021.

\section{Métodos}

El presente estudio está enmarcado en la investigación de enfoque cuantitativo y de alcance descriptivo con un diseño no experimental transversal. La población está constituida por 12 revistas odontológicas venezolanas actuales, con publicaciones fotográficas extraorales en los últimos 11 años, tomando en cuenta los siguientes criterios de inclusión:

1. Revistas odontológicas que se encuentren actualizadas

2. Revistas odontológicas con publicación de casos clínicos con fotografías clínicas extraorales en el periodo 2010 - 2021

3. Revistas odontológicas online con descarga gratuita PDF

Se eligió una muestra no probabilística a conveniencia de revistas odontológicas venezolanas que cumplieron con los criterios de inclusión antes señalados. De esta forma, la muestra estuvo constituida por 6 revistas odontológicas venezolanas con publicaciones de casos clínicos en el periodo 2010 - 2021.
Se empleó el análisis documental como técnica de recolección de datos, a partir de una lista de cotejo como instrumento (Tabla 1), conformada por 6 ítems enumerados del 1 al 6 y sub-ítems representados por letras, que permitieron un análisis sistemático de las normativas editoriales, la preservación de identidad del paciente y consentimiento informado, así como mención de una autorización para el registro y divulgación de las fotografías extraorales en las revistas odontológicas venezolanas. Dicho instrumento fue sometido a juicio de 3 expertos en el área de investigación odontológica. Esta validación consistió en enviar a expertos de destacada trayectoria en investigación odontológica el instrumento de recolección de información junto con una planilla de validación en la cual se evaluaba ítem por ítem de la lista de cotejo, corroborando si se correspondía con los objetivos de la investigación. Una vez realizadas las sugerencias realizadas por los expertos, se enviaron para ser cotejadas y aceptadas por los mismos, dando por finalizado el proceso de validación del instrumento.

Para el análisis de los datos obtenidos, se midieron las variables cualitativas nominales dicotómicas utilizando estadística descriptiva y distribución de frecuencias, para luego ser representados mediante tablas y gráficos realizados por el paquete estadístico SPSS 19 (IBM $®$,

Tabla 1. Instrumento de recolección de información

\begin{tabular}{|c|c|c|c|}
\hline \multicolumn{4}{|c|}{ Criterios de publicación de revistas odontológicas venezolanas } \\
\hline Revista & & Título & \\
\hline Año: & & Vol/Nro & \\
\hline \multicolumn{4}{|c|}{ Exigencia en protocolo de publicación de casos clínicos en la revista } \\
\hline Item & & Si & No \\
\hline 1 & Consentimiento informado & & \\
\hline 2 & Declaración de Helsinki & & \\
\hline 3 & Preservación de identidad del paciente & & \\
\hline \multicolumn{4}{|c|}{ Presentación de caso clínico en la publicación } \\
\hline & & Si & No \\
\hline 4 & Fotografía extraoral & & \\
\hline 5 & Preservación de identidad: & & \\
\hline A & Anonimato & & \\
\hline B & Alteración de la información & & \\
\hline C & Enmascaramiento facial & & \\
\hline 5.1 & Enmascaramiento facial & & \\
\hline A & Banda & & \\
\hline B & Puntos & & \\
\hline $\mathrm{C}$ & Recorte de fotografía extraoral & & \\
\hline $\mathrm{D}$ & Otros & & \\
\hline 6 & Consentimiento informado: & & \\
\hline $\mathrm{A}$ & Uso del consentimiento informado & & \\
\hline a.1 & Publicación de fotografías & & \\
\hline a. 2 & Uso de caso clínico & & \\
\hline a.3 & Ambos & & \\
\hline
\end{tabular}


USA) a fin de determinar la presencia o ausencia de rasgos predominantes.

\section{Resultados}

En la Tabla 2 se muestran 6 revistas en las cuales, destaca la revista Acta Odontológica Venezolana por presentar la mayoría de los artículos publicados. En contraparte, la revista IADR cuenta con el menor número de publicaciones de casos clínicos realizadas en el periodo evaluado.

Tomando en cuenta los criterios para la publicación de fotografías extraorales en las revistas odontológicas venezolanas, 4 de las 6 revistas en estudio requieren dicho documento para el uso del artículo.

Sin embargo, en la Figura 1 se evidencia contradicción de la normativa para la publicación de fotografías en las revistas odontológicas venezolanas, con respecto al uso del consentimiento informado en casos clínicos por parte de los investigadores. Encontrando que la ausencia del consentimiento informado fue representada por casi un tercio de las publicaciones estudiadas.

De este modo, en la Tabla 3 se observa en detalle la frecuencia del uso del consentimiento informado entre las revistas estudiadas. Obteniendo que Acta Odontológica Venezolana no afirma contar con el consentimiento informado, representando más de la mitad de la ausencia del consentimiento informado. Por otro lado, IADR asevera en la totalidad de sus casos clínicos tener el documento.

Al emplear el consentimiento informado, el clínico tiene el deber de comunicar al paciente sobre el mismo, detallando datos de interés sobre el procedimiento clínico

Tabla 2. Publicaciones en revistas odontológicas venezolanas

\begin{tabular}{lcc}
\hline Revistas odontológicas & n & \% \\
\hline ODOUS & 9 & 10,98 \\
Acta Bioclínica & 7 & 8,54 \\
Acta Odontológica Venezolana & 49 & 59,76 \\
Revista Odontológica de los Andes & 7 & 8,54 \\
IADR & 3 & 3,66 \\
Ciencia Odontológica LUZ & 7 & 8,54 \\
\hline
\end{tabular}

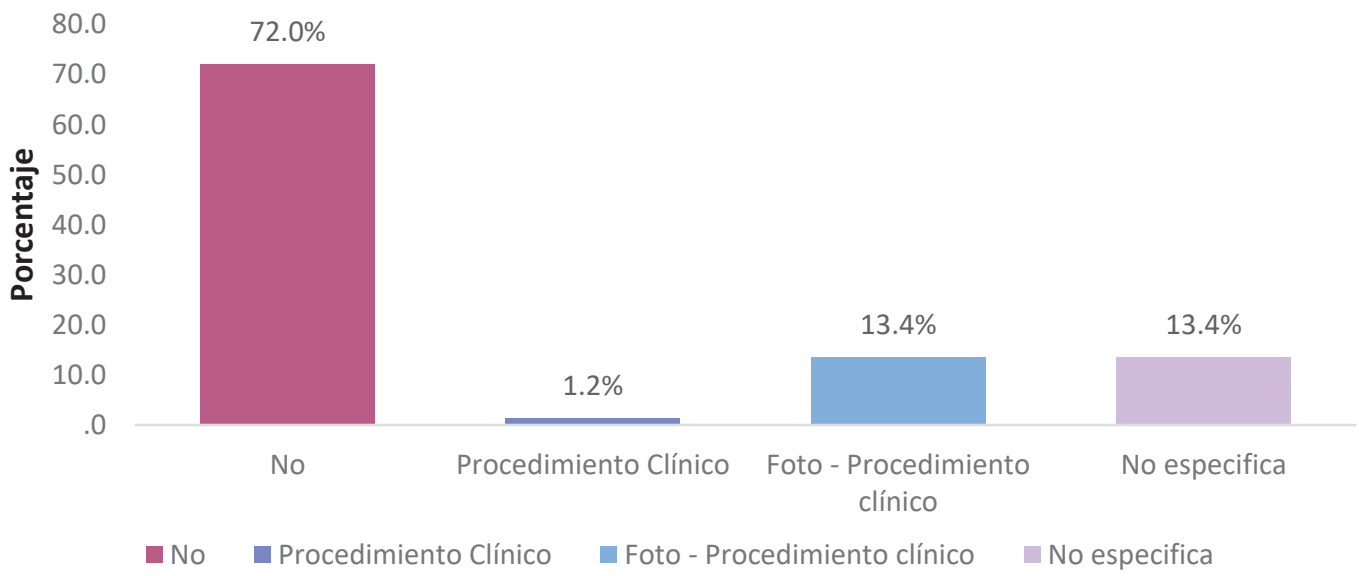

Figura 1. Uso del consentimiento informado

Tabla 3. Uso del consentimiento informado en revistas odontológicas venezolanas

\begin{tabular}{|c|c|c|c|c|c|c|c|c|c|c|c|c|c|c|}
\hline \multirow{3}{*}{$\begin{array}{l}\text { Consentimiento } \\
\text { informado }\end{array}$} & \multicolumn{14}{|c|}{ Revistas odontológicas venezolanas } \\
\hline & \multicolumn{2}{|c|}{ ODOUS } & \multicolumn{2}{|c|}{$\begin{array}{c}\text { Acta } \\
\text { Bioclínica }\end{array}$} & \multicolumn{2}{|c|}{$\begin{array}{l}\text { Acta Odontológica } \\
\text { Venezolana }\end{array}$} & \multicolumn{2}{|c|}{$\begin{array}{l}\text { Revista Odontológica } \\
\text { de los Andes }\end{array}$} & \multicolumn{2}{|c|}{ IADR } & \multicolumn{2}{|c|}{$\begin{array}{c}\text { Ciencia Odontológica } \\
\text { LUZ }\end{array}$} & \multicolumn{2}{|c|}{ Total } \\
\hline & $\mathbf{n}$ & $\%$ & $\mathbf{n}$ & $\%$ & $\mathbf{n}$ & $\%$ & n & $\%$ & $\mathbf{n}$ & $\%$ & $\mathbf{n}$ & $\%$ & $\mathbf{n}$ & $\%$ \\
\hline No & 3 & 3,70 & 4 & 4,90 & 43 & 52,40 & 5 & 6,10 & 0 & 0 & 4 & 4,90 & 59 & 72 \\
\hline $\mathrm{Si}$ & 6 & 7,30 & 3 & 3,70 & 6 & 7,30 & 2 & 2,40 & 3 & 3,70 & 3 & 3,70 & 23 & 28 \\
\hline Total & 9 & 11 & 7 & 8,50 & 49 & 59,80 & 7 & 8,50 & 3 & 3,70 & 7 & 8,50 & 82 & 100 \\
\hline
\end{tabular}


y el uso de su información por parte del investigador. De este modo, en la tabla 3 se obtiene que un bajo porcentaje de los artículos estudiados que afirman tener el consentimiento informado cuentan con la aprobación del paciente para el procedimiento clínico y la toma de fotografía clínica extraoral. En contraparte, la minoría de los artículos aseveran contar con la autorización firmada únicamente para los procedimientos clínicos a realizar.

Asimismo, considerando las diferentes estrategias empleadas para la preservación de identidad del paciente, se determinó la exigencia de la revista para la publicación de fotografías extraorales, observando de este modo que el $50 \%$ de las revistas demandan en sus normas editoriales el uso de métodos que eviten la identificación del paciente en las fotografías extraorales.

$\mathrm{Al}$ analizar la preservación de identidad del paciente en las publicaciones realizadas por las revistas, se obtuvo que solo la Revista Acta Odontológica Venezolana revela la identidad del paciente en una de sus publicaciones, siendo identificable información personal de la paciente ${ }^{39}$, al igual que en la publicación de Acta Bioclínica donde no se enmascaró correctamente al paciente ${ }^{40}$. Sin embargo, el uso de estrategias para preservar el anonimato del paciente son empleadas por la mayoría de los casos clínicos.
En relación con la estrategia de elección para la preservación de identidad (Tabla 4), el anonimato sumado al enmascaramiento facial del paciente es el método de elección en las publicaciones odontológicas, representado por más de la mitad de las publicaciones de los casos clínicos. Por otra parte, un poco más de un tercio de las publicaciones solo emplean el anonimato del paciente como estrategia para la publicación de fotografías extraorales dejando en evidencia el rostro del paciente.

El enmascaramiento facial puede ser realizado con diferentes estrategias (Figura 2), siendo de preferencia para la publicación de fotografías en casos clínicos, el uso de una banda negra en los ojos del paciente. Por otra parte, se utilizó como estrategia de preservación de identidad recortar parte del rostro del paciente para entorpecer su identificación. Asimismo, la minoría de las publicaciones emplean otros métodos como cuadros separados o distorsión de la fotografía a nivel de los ojos del paciente.

\section{Discusión}

Los datos obtenidos en la investigación revelan información de interés sobre los criterios de publicación de las revistas odontológicas venezolanas, describiendo la política editorial, confidencialidad y seguridad. Para obtener estos datos, se identificó el cumplimiento de las

Tabla 4. Uso de estrategias para la preservación de identidad del paciente en las revistas odontológicas venezolanas

\begin{tabular}{|c|c|c|c|c|c|c|c|c|c|c|c|c|c|c|}
\hline \multirow{3}{*}{$\begin{array}{l}\text { Estrategia de } \\
\text { preservación }\end{array}$} & \multicolumn{14}{|c|}{ Revistas odontológicas venezolanas } \\
\hline & \multicolumn{2}{|c|}{ ODOUS } & \multicolumn{2}{|c|}{$\begin{array}{c}\text { Acta } \\
\text { Bioclínica }\end{array}$} & \multicolumn{2}{|c|}{$\begin{array}{l}\text { Acta Odontológica } \\
\text { Venezolana }\end{array}$} & \multicolumn{2}{|c|}{$\begin{array}{l}\text { Revista Odontológica } \\
\text { de los Andes }\end{array}$} & \multicolumn{2}{|c|}{ IADR } & \multicolumn{2}{|c|}{$\begin{array}{c}\text { Ciencia Odontológica } \\
\text { LUZ }\end{array}$} & \multicolumn{2}{|c|}{ Total } \\
\hline & $\mathbf{n}$ & $\%$ & $\mathbf{n}$ & $\%$ & $\mathbf{n}$ & $\%$ & n & $\%$ & $\mathbf{n}$ & $\%$ & $\mathbf{n}$ & $\%$ & $\mathbf{n}$ & $\%$ \\
\hline Ninguna & 0 & 0 & 0 & 0 & 1 & 1,20 & 0 & 0 & 0 & 0 & 0 & 0 & 1 & 1,20 \\
\hline Anonimato & 2 & 2,40 & 2 & 2,40 & 15 & 18,30 & 5 & 6,10 & 1 & 1,20 & 0 & 0 & 25 & 30,50 \\
\hline $\begin{array}{l}\text { Anonimato y } \\
\text { Enmarascamiento }\end{array}$ & 7 & 8,50 & 5 & 6,10 & 33 & 40,20 & 2 & 2,40 & 2 & 2,40 & 7 & 8,50 & 56 & 68,30 \\
\hline Total & 9 & 11 & 7 & 8,50 & 49 & 59,80 & 7 & 8,50 & 3 & 3,70 & 7 & 8,50 & 82 & 100 \\
\hline
\end{tabular}

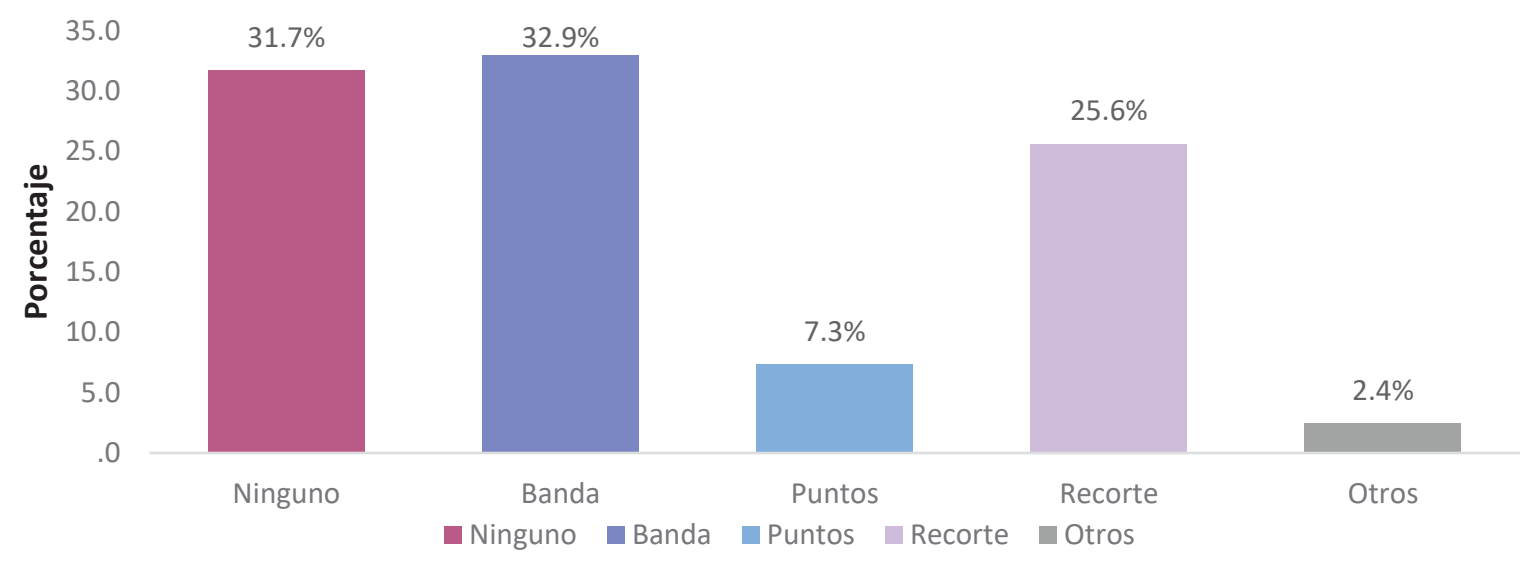

Figura 2. Estrategia de enmascaramiento facial 
normativas éticas por parte de las revistas, en su exigencia de las leyes de bioética, obteniendo que más de la mitad de las revistas estudiadas incluyen en su requerimiento de publicación el desempeño de dichas normas. Concordando con el estudio de Smith ${ }^{41}$, que sugiere que las políticas editoriales deben coincidir con las intenciones de los editores siendo publicadas en una guía para los autores, con el fin de que estos últimos tengan un pensamiento más crítico sobre los detalles esenciales para la descripción de los casos y que estas publicaciones pueden revelar inadvertidamente más información de la que pretenden.

Adicionalmente, se observa que dentro de las políticas editoriales de las seis revistas odontológicas venezolanas se exige el uso del consentimiento informado con el manuscrito previo a la publicación de las fotografías extraorales del paciente en cuatro revistas, lo que representa el $66,7 \%$. En contraste con el estudio realizado por Sardenberg et al. ${ }^{33}$ en Brasil, donde se determinó que de 44 revistas odontológicas solo 3 presentaron orientaciones éticas (Comité de Ética, Declaración del Helsinki, consentimiento del paciente, estándares éticos, requisitos uniformes y sin orientación ética), dos de ellas fueron evaluadas por comisión de ética y uno siguió los estándares éticos.

De acuerdo con la presencia del consentimiento informado en las publicaciones de las revistas odontológicas estudiadas, el 28\% de los casos clínicos afirman tener dicho documento en su artículo, de los cuales, el 13,4\% especifica su uso para la publicación del caso clínico y fotografías extraorales. Así mismo, un artículo (1,2\%) alega tenerlo para el uso de las fotografías del paciente. En contraparte, Bhangoo et al. ${ }^{24}$ en su estudio, aseveran tener un mayor uso del consentimiento informado, demostrando que 43 de 53 departamentos evaluados obtuvieron la autorización de manera verbal y en 10 de forma escrita. Del mismo modo, afirman que el uso de la fotografía clínica ya sea con fines educativos, clínicos o médico legales puede plantear problemas de consentimiento y confidencialidad, y recomiendan la implementación de dicho documento ante la atención médica. Berle ${ }^{42}$, por su parte, afirma que la fotografía es una invasión a la privacidad, sobre todo la fotografía clínica ya que revela particularidades que los pacientes preferirían tener en secreto y aclara que los profesionales de la fotografía clínica deben estar conscientes de esto, $y$ en consecuencia no fotografiar a un paciente a menos que el de su consentimiento.

Por otro lado, se evidenció que el $72 \%$ de los casos clínicos publicados por revistas odontológicas venezolanas no indican en el texto contar con el consentimiento informado. Específicamente, la revista Acta Odontológica Venezolana arrojó que 43 de sus 49 casos clínicos publicados no indicaron el uso de este documento. Esto coincide con el estudio de Stieber et al. ${ }^{21}$, quienes determinaron que si bien se conoce que los pacientes deben proporcionar el consentimiento informado para la publicación de material fotográfico donde sean altamente identificados o no, no se encontró un consenso sobre los requerimientos de las revistas a los autores, ya que existen revistas que solicitan el consentimiento informado por escrito, mientras otras solo requieren que los autores certifiquen que lo obtuvieron. En el mismo sentido, establecen que deben incluir el propósito de las fotografías, el medio por el cual serán mostradas, como se protegerá la identidad del paciente, y en caso de que la fotografía se publique en internet deben solicitar un consentimiento específico para su divulgación electrónica.

En cuanto a la autorización para divulgación de los resultados, este estudio encontró que $13,4 \%$ de los casos clínicos con fotografías extraorales que presentaban el consentimiento informado del paciente, dieron a conocer que, en este, se solicitaba la autorización para la publicación de su caso y fotografía clínica. Esto concuerda con el estudio de Lau et al. ${ }^{43}$, quien expone que el 55\% de los pacientes estuvieron de acuerdo con la publicación de fotografías clínicas extraorales identificables dentro del consentimiento informado. De este modo, Palacios-Gonzáles ${ }^{44}$ en su investigación, afirma que para el uso de la fotografía clínica extraoral el paciente debe ser informado sobre el propósito de las mismas, describiendo así, dos niveles de información dentro del consentimiento. El primer nivel, será limitado al tratamiento del paciente, mientras que el segundo incluye publicación de la información y su uso para fines académicos. Concordando con Segal ${ }^{29}$, quien asevera que el consentimiento debe describir todos los usos de las fotografías, ya sea para el registro médico, fines educativos o publicación.

En cuanto al ítem de preservación de identidad, las políticas de editorial de las revistas odontológicas venezolanas demandan el uso de métodos que permitan resguardar la integridad del paciente y cumplir los requisitos éticos de la investigación. Para esto, se determinó la exigencia de las revistas en cuanto al anonimato del paciente a partir de estrategias como el recorte de la fotografía extraoral, un recuadro negro en los ojos y difuminar la imagen para dificultar su reconocimiento. De esta manera, el $50 \%$ de las revistas exigen para la publicación de artículos el uso de diversas estrategias, a fin de preservar la identidad del paciente.

En contraparte, Shintani et al. ${ }^{30}$ obtuvo que el $60 \%$ de las revistas que evaluaron mostraban la totalidad del rostro del paciente en su publicación, y en el caso de emplear enmascaramiento facial, este era inadecuado o insuficiente. Si bien parece alarmante, los números solo reflejan la importancia que le otorgan las revistas al consentimiento informado por encima del enmascaramiento facial. Asimismo, la investigación demostró que el uso de enmascaramiento se debía a la necesidad de adicionar anonimato, y otorgar privacidad al paciente a pesar de obtener el consentimiento informado.

En este sentido, McKelvie ${ }^{45}$ considera que, si bien el uso del enmascaramiento facial es una estrategia importante para preservar la identidad del paciente, no es el ideal, ya que no es efectiva en su totalidad porque aun con el método de preservación de identidad del paciente este es identificable. Esto concuerda con los resultados de 
la presente investigación, debido a que el $68,3 \%$ de los artículos publicados usan el enmascaramiento facial sumado al anonimato del paciente como estrategias para la publicación de fotografías extraorales, con la intención de obtener mejores resultados y preservar la identidad del paciente. Sin embargo, 30,4\% de los casos clínicos estudiados, arrojaron el anonimato como método de elección único, resaltando la importancia del consentimiento informado para la publicación de las fotografías en casos clínicos.

Adicionalmente, Roberts et al. ${ }^{31}$ relata la discusión de diferentes investigaciones acerca de la ética en la fotografía clínica, así como también la efectividad de la desidentificación mediante métodos tradicionales, como barras en los ojos las cuales previenen la identificación del paciente tanto como lo hacen unos lentes oscuros. Por lo tanto, la colocación de barras, círculos u otro elemento en el área de los ojos de la fotografía, no es considerada para este autor como un método eficiente para disuadir la identificación del sujeto.

En cuanto al ítem de la estrategia de enmascaramiento facial de elección por parte de los investigadores para la publicación de fotografías extraorales, se obtuvo que 27 publicaciones tienen predilección por el uso de una banda negra en los ojos del paciente y 21 publicaciones por el recorte de la fotografía. En contraparte, 26 de las publicaciones no tienen métodos para ocultar la identidad del paciente. Nylenna et al. ${ }^{32}$ en su estudio, evaluaron 75 publicaciones, de las cuales 17 aceptaban realizar modificaciones fáticas, siendo su principal elección para la preservación de identidad del paciente, por encima de las modificaciones fotográficas para disuadir el reconocimiento. En contraste, Engelstad et al. ${ }^{46}$ determinaron que existe un patrón holístico de reconocimiento, es decir, que cuando se está advertido de la existencia de un rostro familiar, el individuo hará una evaluación detallada a fin de realizar el reconocimiento, asimismo, afirma que el uso de estrategias de preservación de identidad pueden afectar directamente la estética y calidad diagnostica de la fotografía, dejando de ser estas fundamentales para el anonimato del paciente siempre y cuando se obtenga el consentimiento informado por escrito.

En concordancia con lo expresado anteriormente, Franchitto et al. ${ }^{47}$, asevera que existen muchas maneras de proteger el anonimato del paciente, sin embargo es inadecuado y está en desuso, debido a que marcas como tatuajes, cicatrices y joyería pueden estar visibles y hacen posible la identificación. De esta forma, obtener la garantía del consentimiento informado permite respetar la privacidad y autonomía del paciente.

De forma similar, Bennett et al. ${ }^{22}$, presentan una guía de publicaciones éticas de fotografía facial donde recomiendan el uso del consentimiento informado para las fotografías extraorales de manera individual según sea el uso (para conferencias, educación, publicación, y redes sociales) donde se ofrezca la oportunidad al paciente de aprobar las fotos en las que puede ser identificado, ya que el enmascaramiento facial es insuficiente para disuadir su identificación. Del mismo modo, re- comiendan evitar publicar información personal, fechas, número de historia, localización y nombres en la fotografía publicada.

Cabe destacar que, al no indicar tener el consentimiento informado, errores en la técnica de preservación de identidad del paciente puede conllevar al incumplimiento de las condiciones para el uso de la fotografía clínica extraoral. Permitiendo así que ante el uso ineficaz de métodos sea reconocible la imagen del individuo, tal como se muestra en la publicación de la revista Acta Bioclínica cuyo documento PDF no tiene una adecuada alineación entre los círculos y los ojos de la misma como estrategia de preservación de la identidad ${ }^{40}$.

Finalmente, hay estudios que señalan que, en vez de llevar a cabo la práctica inadecuada del enmascaramiento facial, es necesario solicitar el consentimiento informado al paciente para la toma y uso de fotografías, bien puedan ser identificados o no. Esto garantiza el respeto a la privacidad del paciente y su autonomía ${ }^{21,48}$. Sin embargo, es importante resaltar que este estudio se vio limitado por la falta de actualización de las revistas odontológicas venezolanas, lo que impidió que fueran abarcadas en su totalidad, disminuyendo la muestra/población de nuestra investigación. Además, la no uniformidad de criterios en las revistas acerca de la publicación de las fotografías extraorales dificultó la identificación de métodos de preservación de identidad. Así como la falta de especificidad en el consentimiento informado impidió verificar la inclusión del permiso de publicación de fotografías clínicas extraorales en este.

Se concluye que la revista Acta Odontológica fue la que más utilizó el anonimato y el enmascaramiento como método de preservación de la identidad. El método que se empleó con mayor frecuencia fue la banda para cubrir los ojos. De igual forma, el empleo del consentimiento informado fue utilizado con mayor frecuencia por la revista Acta Odontológica.

El uso del consentimiento informado permite descartar el uso de estrategias de preservación de identidad del paciente, con el fin de mejorar la finalidad diagnóstica y estética de la fotografía.

Se hace importante, realizar la mención explícita del uso del consentimiento informado dentro del texto, por lo cual se recomienda a los comités editoriales de revistas odontológicas venezolanas a velar con el cumplimiento de normativas internacionales para brindar una mayor protección al involucrado en el reporte de caso y a los autores del mismo, lo cual se traduce en elevar la calidad de los manuscritos desde el punto de vista bioético.

\section{Referencias bibliográficas}

1. Roa R, Miranda S, Chidiak R, Moreno M, Rodriguez AM. Selección y configuración de la cámara digital para fotografía clínica. Parte 1: Fotografía clínica extraoral. Rev Odontol Los Andes [Internet]. 2007 [citado el 03 de julio de 2021]; 2(1):71-8. Disponible en: https://www. semanticscholar.org/paper/Selecci\%C3\%B3n-y-configuraci $\% \mathrm{C} 3 \% \mathrm{~B} 3 \mathrm{n}$-de-la-c\%C3\% 1 mara-digi- 
tal-para-Revisi\%C3\%B3n/be2f5319b232fccc7d2916dc395162670fb158ce.

2. Kwak JH, Chen E. An overview of the american board of orthodontics certification process. APOS Trends Orthod. 2018;8 (1):14-20. DOI:10.4103/apos218.

3. American Board Orhodontics. Ideal photographs and radiographs. American Board of Orthodontics. [Citado el 07 de julio de 2021]. Disponible en: https://www.americanboardortho.com/media/1206/example-photos-radiographs.pdf

4. López L. Eficacia en el diagnóstico odontológico con el uso de la fotografia digital clínica. [tesis de Maestría]. [Lima]: Escuela Universitaria de Posgrado, Universidad Nacional Federico Villarreal; 2018. 77 p.

5. Caicedo CGV, Nuñez JB. Utilización de la fotografía dental como auxiliar de diagnóstico para un correcto tratamiento odontológico en los consultorios privados de la ciudad de san pedro de Riobamba en el período marzo-julio 2014 [tesis de bachiller]. [Riobamba]: Facultad de Ciencias de la Salud, Universidad Nacional de Chimborazo; 2015. 47 p.

6. Yoshio I, Calixto LR. Fotografia odontológica em dentes anteriores-descrição da técnica. RevDental Press Estét [Internet]. 2011; [citado el 05 de julio de 2021]; 8(4):15-22. Disponible en: https://web.b.ebscohost.com/abstract?direct $=$ true \& profile $=$ ehost $\&$ scope $=$ site $\&$ authtype $=$ crawl er\&jrnl $=18072488 \& A N=67517510 \& \mathrm{~h}=\mathrm{x} 4 \mathrm{GhYYLgSz}-$ ruwDt9F18APb1pQbR2U2oiStqqwJ8vCduRhlmKpFUtIcqAtzQr4gLTbRn5MzWjaI\%2b12s8473Q2C$\mathrm{Q} \% 3 \mathrm{~d} \% 3 \mathrm{~d} \& \mathrm{crl}=\mathrm{c} \&$ resultNs=Admin WebAuth\&result Local = ErrCrlNotAuth \&crlhashurl=login. aspx $\% 3$ fdirect $\% 3$ dtrue $\% 26$ profile $\% 3$ dehost $\%$ 26 scope $\% 3$ dsite\%26authtype\%3dcrawler\%26jrn$1 \% 3 \mathrm{~d} 18072488 \% 26 \mathrm{AN} \% 3 \mathrm{~d} 67517510$.

7. Fernandez-Bozal J. Fotografia intraoral y extraoral. Rev Esp Ortod [Internet]. 2006; [citado el 05 de julio de 2021]; 36(1):49-58. Disponible en: http://www.revistadeortodoncia.com/files/2006_36_1_049-058.pdf.

8. McLaren EA, Garber DA, Figueira J. The photoshop smile design technique (part 1): digital dental photography. Compend Contin Educ Dent [Internet]. 2013 [citado el 05 de julio de 2021]; 34(10):772, 774, 776 passim. Disponible en: https://pubmed.ncbi.nlm.nih. gov/24571506/.

9. Fernández-Bozal J. Fotografía digital: ventajas e inconvenientes. Rev Esp Ortod [Internet]. 2004 [citado el 05 de julio de 2021]; 34(4):335-42. Disponible en: https:// dialnet.unirioja.es/servlet/articulo? codigo $=1125935$.

10. Uzunov TT, Kosturkov D, Uzunov T, Filchev D, Bonev B, Filchev A. Application of photography in dental practice. J of IMAB. 2015;21(1):682-6. DOI: 10.5272/ jimab.2015211.682.

11. Wander P, Ireland RS. Dental photography in record keeping and litigation. Br Dent J. 2014;217(3):133-7. DOI: $10.1038 /$ sj.bdj.2014.649.

12. Hein $S$, Zangl $M$. The use of a standardized gray reference card in dental photography to correct the effects of five commonly used diffusers on the color of 40 extracted human teeth. Int J Esthet Dent [Internet]. 2016 [citado el 05 de julio de 2021]; 11(2):246-
59. Disponible en: https://www.researchgate.net/ publication/305476888_The_use_of_a_standardized_gray_reference_card_in_dental_photography_to_ correct_the_effects_of_five_commonly_used_diffusers_on_the_color_of_40_extracted_human_teeth.

13. Mladenović D, Mladenović L, Mladenović S. Importance of digital dental photography in the practice of dentistry. Sci J Fac Med Niš [Internet]. 2010 [citado el 05 de julio de 2021]; 27(2):75-9. Disponible en: https://publisher. medfak.ni.ac.rs/AFMN_1/2010/2-2010/4\%20Dragan\%20Mladenovic.pdf.

14. Ayala JC. Fotografías digitales en la clínica de ortodoncia: conceptos básicos. RV AcBO [Internet]. 2014 [citado el 05 de julio de 2021];3(2):1-37. Disponible en: http://www.rvacbo.com.br/ojs/index.php/ojs/article/ view/188/146.

15. Yoshio I, Calixto LR. Fotografia de face na odontologia. Rev Dental Press Estét [Internet]. 2011 [citado el 05 de julio de 2021]; 8(2):42-50. Disponible en: http://bases. bireme.br/cgi-bin/wxislind.exe/iah/online/?IsisScript=i$\mathrm{ah} /$ iah. .xis\&src $=$ google \&base $=$ LILACS \&lang $=$ p\&nex tAction $=\operatorname{lnk} \&$ exprSearch $=594767$ \&indexSearch=ID.

16. Casaglia A, De Dominicis P, Arcuri L, Gargari M, Ottria L. Dental photography today. Part 1: basic concepts. Oral Implantol (Rome). 2016;8(4):122-9. DOI: 10.11138/orl/2015.8.4.122.

17. Hochman B, Nahas FX, Ferreira LM. Photography in medical research. Acta Cir Bras. 2005;20(Suppl 2):1925. DOI: $10.1590 / s 0102-86502005000800006$.

18. Neiva-Silva L, Koller SH. O uso da fotografia na pesquisa em psicologia. Estud Psicol. 2002;7(2):237-50. DOI: 10.1590/S1413-294X2002000200005.

19. Desai V, Bumb D. Digital dental photography: a contemporary revolution. Int J Clin Pediatr Dent. 2013;6(3):1936. DOI: $10.5005 /$ jp-journals-10005-1217.

20. Solesio Pilarte F, Lorda Barraguer E, Lorda Barraguer A, Laredo Ortiz C, Rubio Verdú R. Estandarización fotográfica en cirugía plástica y estética. Cir Plast Iberolatinoam [Internet]. 2009 [citado el 05 de julio de 2021]; 35(2):79-90. Disponible en: http://scielo.isciii.es/scielo.php?script=sci_arttext\&pid=S0376-78922009000200001\&lng=es.

21. Stieber JC, Nelson T, Huebner CE. Considerations for use of dental photography and electronic media in dental education and clinical practice. J Dent Educ. 2015;79(4):432-8. DOI: $10.1002 / \mathrm{j} .0022-$ 0337.2015.79.4.tb05901.x.

22. Bennett KG, Bonawitz SC, Vercler CJ. Guidelines for the ethical publication of facial photographs and review of the literature. Am Cleft Palate-Craniofacial J. 2019;56(1):7-14. DOI: 10.1177/1055665618774026.

23. Bernardes MJ, Nunes R. Consentimento dos doentes para a publicação de casos clínicos: Revisão da literatura. Arq Med. 2013;27(2):58-64. DOI: 10.1016/j.reuma.2018.07.007.

24. Bhangoo P, Maconochie IK, Batrick N, Henry E. Clinicians taking pictures a survey of current practice in emergency departments and proposed recommendations of best practice. Emerg Med J. 2005;22(11):761-5. DOI: 10.1136/emj.2004.016972. 
25. Taylor D, Foster E, Dunkin C, Fitzgerald A. A study of the personal use of digital photography within plastic surgery. J Plast Reconstr Aesthetic Surg. 2008;61(1):3740. DOI: 10.1016/j.bjps.2007.02.033.

26. Green BN, Johnson CD. How to write a case report for publication. J Chiropr Med. 2006;5(2):72-82. DOI: 10.1016/S0899-3467(07)60137-2.

27. Henley LD, Frank DM. Reporting ethical protections in physical therapy research. Phys Ther. 2006;86(4):499509. DOI: $10.1093 / \mathrm{ptj} / 86.4 .499$.

28. Vallejo Becerra D. Fotografía en odontología. Rev Estomatol. 2017;9(1):33-8. DOI: 10.25100/re.v9i1.5508.

29. Segal J, Sacopulos MJ. Photography consent and related legal issues. Facial Plast Surg Clin North Am. 2010;18(2):237-44. DOI: 10.1016/j.fsc.2010.01.003.

30. Shintani M, Williams J. Need for greater consensus on protection of patient anonymity and rights in facial photographs: a survey of international and domestic oral surgery journals. Bull Tokyo Dent Coll. 2012;53(4):20712. DOI: $10.2209 /$ tdcpublication.53.207.

31. Roberts EA, Troiano C, Spiegel JH. Standardization of guidelines for patient photograph deidentification. Ann Plast Surg. 2016;76(6):611-4. DOI: 10.1097/ SAP.0000000000000817.

32. Nylenna M, Riis P. Identification of patients in medical publications: need for informed consent. Br Med J. 2009;302(6786):1182. DOI: 10.1136/ bmj.302.6786.1182.

33. Sardenberg T, Müller SS, Pereira HR, Oliveira RA, Hossne WS. Análisis de los aspectos éticos de la investigación en seres humanos contenidos en las instrucciones a los autores de 139 revistas científicas brasileñas. Acta Bioeth. 2000;6(2):293-307. DOI: 10.4067/S1726569X2000000200008.

34. Mancini RR, Lolas SF. Evaluacion bioética de trabajos de investigacion en seres humanos publicados en America Latina y el Caribe. Acta Bioeth. 2001;7(1):159-69. DOI: 10.4067/S1726-569X2001000100012.

35. Schroter S, Plowman R, Hutchings A, Gonzalez A. Reporting ethics committee approval and patient consent by study design in five general medical journals. J Med Ethics. 2006;32(12):718-23. DOI: 10.1136/ jme.2005.015115.

36. Sumathipala A, Siribaddana S, Hewege S, Lekamwattage M, Athukorale M, Siriwardhana C, et al. Ethics review committee approval and informed consent: an analysis of biomedical publications originating from Sri Lanka. J Biomed Sci. 2008;9(3):1-7. DOI: 10.1186/1472-69399-3.

37. Elsayed DEM, Elamin RM. Documentation of ethical considerations in published articles in Sudanese medical journals. South African J Bioeth Law [Internet]. 2009 [citado el 05 de julio 2021]; 2(1):32-4. Disponible en:
file:///Users/rossemary/Downloads/50242-Article $\% 20$ Text-72186-1-10-20100129.pdf.

38. Bernardes MJ, Nunes R. Consentimento dos doentes para a publicaçáo de casos clínicos: Revisão da literatura. Arq Med. 2013;27(2):58-64. DOI: 10.1016/j.reuma.2018.07.007.

39. Pimentel HE. Alergia al látex en una profesional de la odontología. Presentación de caso clínico. Acta Odontol Venez [Internet]. 2015 [citado el 05 de julio de 2021]; 53(1):1-7. Disponible en: https://www.actaodontologica.com/ediciones/2015/1/art-8/.

40. Pulido ES, Dulcey VC, Villarroel-Dorrego M, Carrera C, Frías JI, Pérez RR, et al. Síndrome autoinmune/inflamatorio inducido por adyuvantes: Reporte de un caso. Acta Bioclin [Internet]. 2018 [citado el 05 de julio de 2021]; 8(15):204-23. Disponble en: http://erevistas.saber.ula. ve/index.php/actabioclinica/article/view/9967/9894.

41. Smith J. Keeping confidences in published papers. BMJ. 1991;302(6786):1168. DOI: 10.1136/ bmj.302.6786.1168.

42. Berle I. Clinical photography and patient rights: The need for orthopraxy. J Med Ethics. 2008;34(2):89-92. DOI: $10.1136 /$ jme.2006.019166.

43. Lau CK, Schumacher HHA, Irwin MS. Patients' perception of medical photography. J Plast Reconstr Aesthet Surg. 2010;63(6):e507-11. DOI: 10.1016/j. bjps.2009.11.005.

44. Palacios-Gonzalez C. The ethics of clinical photography and social media. Med Health Care Philos. 2015;18(1):63-70. DOI: 10.1007/s11019-014-9580-y.

45. McKelvie SJ. The role of eyes and mouth in the memory of a face. Am J Psychol. 1976;89(2):311-23. DOI: $10.2307 / 1421414$

46. Engelstad ME, McClellan M, Jacko JA, Melton GB. Deidentification of facial images using composites. J Oral Maxillofac Surg. 2011;69(12):3026-31. DOI: 10.1016/j.joms.2011.01.011.

47. Franchitto N, Gavarri L, Dédouit F, Telmon N, Rougé D. Photography, patient consent and scientific publications: medicolegal aspects in France. J Forensic Leg Med. 2008;15(4):210-2. DOI: 10.1016/j.jflm.2007.08.004.

48. Rodríguez M, Linares Cano MA, Ortega Pertuz AI, Perez L, Romero L. Ameloblastoma desmoplásico en parasínfisis mandibular: reporte de un caso. ODOUS Cientifica [Internet]. 2014 [citado el 05 de julio de 2021]; 15(2):37-43. Disponible en: https://biblat.unam.mx/ hevila/ODOUSCientifica/2014/vol15/no2/5.pdf. 